\title{
Differential transform method to solve two-dimensional Volterra integral equations with proportional delays
}

\author{
Şuayip Yüzbaşi and Nurbol Ismailov \\ Department of Mathematics, Faculty of Science, Akdeniz University, TR 07058 Antalya, Turkey
}

Received: 7 March 2016, Accepted: 4 May 2016

Published online: 25 October 2017.

\begin{abstract}
In this paper, the differential transform method is extended by providing a new theorem to two-dimensional Volterra integral equations with proportional delays. The method is useful for both linear and nonlinear equations. If solutions of governing equations can be expanded for Taylor series, then the method gives opportunity determine coefficients Taylor series, i.e. the exact solutions are obtained in series form. In illustrate examples the method applying to a few type equations.
\end{abstract}

Keywords: Two-dimensional Volterra integral equations with proportional delays, partial differential equations, differential transform method.

\section{Introduction}

In 1897 by Vito Volterra [1] considered integral equations which limits of integration variable and limits represents a proportional delays vanishing at $t=0$. Volterra preceded the analysis of the existence and uniqueness of the solution. In 1927 and 1937 papers, on population dynamics Volterra studied integro-differential equations with delays. We can see plenty of monographs and papers devoted for Volterra functional equations and their applications. For example, the nonlinear Volterra integral and integro-differential equations with delays are described models in epidemiology and population growth $[2,3,4,5,6,7,8]$.

There are many authors has studied numerical analysis of Volterra integral and integro-differential equations, for example, the collocation methods for Volterra integral and integro-differential equations with proportional delays were first studied in detail in Brunner [9], Zhang [11], Takama [10], Bellen [12]. Yüzbaşı [13] has applied Laguerre polynomials for pantograph-type Volterra integro-differential equations. The systems of Volterra integral equations with variable coefficients has been solving by Bessel polinomials in [14]. In addition, the homotopy perturbation method [15], the variational iteration method [16], the Galerkin method [17], the Adomian decomposition method [18] and theirs various modified methods has been used for solving above mentioned equations.

In this paper, we consider the two-dimensional Volterra integral equations with proportional delays the following forms:

$$
u(x, t)=f(x, t)+g(x, t),
$$

\footnotetext{
*Corresponding author e-mail: syuzbasi@akdeniz.edu.tr
} 
where

$$
f(x, t)=\left\{\begin{array}{l}
\int_{0}^{r_{1} t} \int_{0}^{r_{2} x} h(y, z) u(p y, q z) d y d z \\
\int_{0}^{r_{1} t} \int_{0}^{r_{2} x} \frac{u(p y, q z)}{v(y, z)} d y d z \\
\frac{1}{v(x, t)} \int_{0}^{r_{1} t \int_{0}^{r_{2} x} u(p y, q z) d y d z}
\end{array}\right.
$$

where $r_{1}, r_{2}, p, q \in(0,1], h, f, v$ are given functions.

The rest of this paper is arranged as follows. In section 2, the fundamental relations and two theorems are given for two-dimensional differential transform method. In Section 3, we extend the differential transform method by the new theorem for two-dimensional Volterra integral equations with proportional delays. We apply this method to some two-dimensional Volterra integral equations with proportional delays in Section 4. Section 5 concludes this study with a brief summary.

\section{Two-dimensional differential transform}

The differential transform method is presented by Pukhov [19] and Zhou [20] in study of electric circuits. The main idea of method is transformed the given functional equations to difference equations, and by using initial conditions calculate the values of derivatives of functions at given point. In recent years, the method have been applying a large class of problems, in particular, Tari et al. [21,22] and Jang [23] are applied for two-dimensional Volterra integro-differential equations.

Suppose a function $u(x, t)$ is analytic in the given domain $D$ and $\left(x_{0}, t_{0}\right) \in D$.

Definition 1. The two-dimensional differential transform of function $u(x, t)$ at $\left(x_{0}, t_{0}\right)$ is defined as following

$$
U(n, m)=\frac{1}{n ! m !}\left[\frac{\partial^{n+m} u(x, t)}{\partial x^{n} \partial t^{m}}\right]_{\substack{x=x_{0} \\ t=t_{0}}}
$$

Definition 2. Differential inverse transform of $U(n, m)$ is defined as

$$
u(x, t)=\sum_{n=0}^{\infty} \sum_{m=0}^{\infty} U(n, m)\left(x-x_{0}\right)^{n}\left(t-t_{0}\right)^{m} .
$$

From the definitions differential transform and differential inverse transform it is easy to obtain the following theorems.

Theorem 1. Assume that $U(n, m)$ and $U_{i}(n, m)(i=1,2)$ are the two-dimensional differential transforms of the functions $u(x, t)$ and $u_{i}(x, t)$ at $(0,0)$ respectively, then

If $u(x, t)=a u_{1}(x, t) \pm b u_{2}(x, t)$, then $U(n, m)=a U_{1}(n, m) \pm b U_{2}(n, m)$, a and $b$ are real numbers.

If $u(x, t)=u_{1}(x, t) u_{2}(x, t)$, then $U(n, m)=\sum_{l=0}^{m} \sum_{k=0}^{n} U_{1}(k, l) U_{2}(n-k, m-l)$.

If $u(x, t)=\frac{\partial^{k+l} v(x, t)}{\partial^{k} x \partial^{l} t}$, then $U(n, m)=(n+1)(n+2) \ldots(n+k)(m+1)(m+2) \ldots(m+l) V(n+k, m+l)$.

If $u(x, t)=\int_{0}^{t} \int_{0}^{x} v(y, z) d y d z$, then $U(n, 0)=U(0, m)=0, U(n, m)=\frac{1}{n m} V(n-1, m-1)$.

Theorem 2. Assume that $U(n, m)$ and $U_{i}(n, m)(i=1,2)$ are the two-dimensional differential transforms of the functions $u(x, t)$ and $u_{i}(x, t)$ at $(0,0)$ respectively, $p, q, p_{i}, q_{i} \in(0,1]$, then 
If $u(x, t)=v(p x, q t)$, then $U(n, m)=p^{n} q^{m} V(n, m)$.

If $u(x, t)=u_{1}\left(p_{1} x, q_{1} t\right) u_{2}\left(p_{2} x, q_{2} t\right)$, then

$U(n, m)=\sum_{l=0}^{m} \sum_{k=0}^{n} p_{1}^{k} p_{2}^{l} q_{1}^{n-l} q_{2}^{m-l} U_{1}(k, l) U_{2}(n-k, m-l)$.

If $u(x, t)=\frac{\partial^{k+l} v(p x, q t)}{\partial^{k} x \partial^{l} t}$, then $U(n, m)=(n+1)(n+2) \ldots(n+k)(m+1)(m+2) \ldots(m+l) p^{n+k} q^{m+l} V(n+k, m+l)$.

The proofs of Theorems 1-2 can be found in [21,24].

\section{Main results}

In this section, we present the differential transform relations that can be used for solving two-dimensional Volterra integral equations with proportional delays.

Theorem 3. Assume that $F(n, m), U(n, m)$ and $V(n, m)$ are the two-dimensional differential transforms of the functions $f(x, t), u(x, t)$ and $v(x, t)$ at $(0,0)$ respectively, $p, q, r_{1}, r_{2} \in(0,1]$, then:

(a) If $f(x, t)=\int_{0}^{r_{1} t} \int_{0}^{r_{2} x} u(p y, q z) d y d z$, then $F(n, 0)=F(0, m)=0, F(n, m)=\frac{1}{n m} q^{m-1} p^{n-1} r_{2}^{n} r_{1}^{m} U(n-1, m-1)$.

(b) If $f(x, t)=\int_{0}^{r_{1} t} \int_{0}^{t} \frac{u(p y, q z)}{v(y, z)} d y d z$, then $U(n, m)=\frac{1}{p^{n} q^{m}} \sum_{l=0}^{m} \sum_{k=0}^{n} r_{2}^{k-n-1} r_{1}^{l-m-1}(n-k+1)(m-l+1) V(k, l) F(n-k+1, m-$ $l+1)$.

(c) If $f(x, t)=\frac{1}{v(x, t)} \int_{0}^{r_{1} t} \int_{0}^{r_{2} x} u(p y, q z) d y d z$, then $\sum_{l=0}^{m} \sum_{k=0}^{n} V(k, l) F(n-k, m-l)=\frac{1}{n m} r_{1}\left(r_{1} q\right)^{m} r_{2}\left(r_{2} p\right)^{n} U(n-1, m-1)$

Proof. (a) From definition differential transform we have $F(n, 0)=F(0, m)=0, \quad(n, m=0,1,2, \ldots)$. Since

$$
\frac{\partial^{2} f(x, t)}{\partial\left(r_{2} x\right) \partial\left(r_{1} t\right)}=u\left(p r_{2} x, q r_{1} t\right)
$$

from Theorem 1-2 we have

$$
\frac{1}{r_{1} r_{2}}(n+1)(m+1) F(n+1, m+1)=\left(p r_{2}\right)^{n}\left(q r_{1}\right)^{m} U(n, m)
$$

(b) Analogously to part $(a), F(n, 0)=F(0, m)=0,(n, m=0,1,2, \ldots)$. Since

$$
u\left(r_{2} p x, r_{1} q t\right)=\frac{1}{r_{1} r_{2}} v\left(r_{2} x, r_{1} t\right) \frac{\partial^{2} f(x, t)}{\partial x \partial t}
$$

using differential transform of multiplication of functions and Theorem 2, we have the following:

$$
\left(r_{2} p\right)^{n}\left(r_{1} q\right)^{m} U(n, m)=\frac{1}{r_{1} r_{2}} \sum_{l=0}^{m} \sum_{k=0}^{n} r_{2}^{k} r_{1}^{l}(n-k+1)(m-l+1) V(k, l) F(n-k+1, m-l+1), \quad(n, m=1,2, \ldots) .
$$

(c) Sinse $v(x, t) f(x, t)=\int_{0}^{r_{1} t} \int_{0}^{r_{2} x} u(p y, q z) d y d z$, using differential transform for multiplication of functions from Theorem 1 and two-dimensional integral with proportional delays from Theorem 3(a), we get necessary equation.

By using this theorem proved for solving two-dimensional integral equations, two-dimensional integral equations will be solve usefully. 


\section{Illustrate examples}

In this section, using differential transform and relations in Theorem 3, we get solutions in series form of integral equations (1).

Example 1. Let us consider linear two-dimensional Volterra integral equation with proportional delays given by

$$
u(x, t)=x t+2 x t^{2}-\frac{1}{8} x^{2} t^{2}-\frac{1}{6} x^{2} t^{3}+\int_{0}^{t} \int_{0}^{x} u\left(\frac{y}{2}, z\right) d y d z
$$

For this problem, $f(x, t)=\int_{0}^{t} \int_{0}^{x} u\left(\frac{y}{2}, z\right) d y d z$ and $g(x, t)=x t+2 x t^{2}-\frac{1}{8} x^{2} t^{2}-\frac{1}{6} x^{2} t^{3}$.

Using differential transform of equation, we have the following

$$
U(n, m)=\frac{1}{n m 2^{n-1}} U(n-1, m-1)+\delta(n-1, m-1)+2 \delta(n-1, m-2)-\frac{1}{8} \delta(n-2, m-2)-\frac{1}{6} \delta(n-2, m-3),
$$

where $\delta$ is Kroneker symbol and $\delta(n, m)=\delta(n) \delta(m) . U(n, 0)=U(0, m)=0(n, m=0,1,2, \ldots), U(1,1)=1$, $U(1,2)=2$, In other cases $U(n, m)=0$.

Using equation (3) we get the exact solution $u(x, t)=x t+2 x t^{2}$.

Example 2. We consider the following two-dimensional Volterra integral equation with proportional delays where

$$
u(x, t)=e^{x+t}-\frac{3 t}{4}\left(1-e^{-\frac{2}{3} x}\right)+\int_{0}^{\frac{1}{2} t} \int_{0}^{x} \frac{u\left(\frac{y}{3}, z\right)}{e^{y+z}} d y d z
$$

This problem is given by $f(x, t)=\int_{0}^{\frac{1}{2} t} \int_{0}^{x} \frac{u\left(\frac{y}{3}, z\right)}{e^{y+z}} d y d z$ and $g(x, t)=e^{x+t}-\frac{3 t}{4}\left(1-e^{-\frac{2}{3} x}\right)$ in (1). Now, applying differential transform to given equation and using Theorem 3 , we have

$$
U(n, m)=F(n, m)+\frac{1}{n ! m !}-\frac{3}{4} \delta(n, m-1)+\frac{3}{4}\left(-\frac{2}{3}\right)^{n} \frac{1}{n !} \delta(m-1) .
$$

Solving recurrence equations (4), we obtain

$$
\begin{array}{rlrl}
U(0,0) & =F(0,0)+1+0+0 & U(0,2) & =F(0,2)+\frac{1}{2 !}-0+0 \\
U(1,0) & =F(1,0)+1+0-0 & U(1,2)=F(1,2)+\frac{1}{2 !} \\
U(0,1)=F(0,1)+1-\frac{3}{4}+\frac{3}{4} & U(2,1)=F(2,1)+\frac{1}{2 !}+\frac{1}{2} \frac{1}{3} \\
U(1,1)=F(1,1)+1+0-\frac{1}{2} & U(2,2)=F(2,2)+\frac{1}{2 !} \frac{1}{2 !} \\
U(2,0)=F(2,0)+\frac{1}{2 !}-0+0 & U(3,1)=F(3,1)+\frac{1}{3 ! 2 !}+\frac{3}{4}\left(-\frac{2}{3}\right)^{3} \frac{1}{3 !}
\end{array}
$$


where $F(n, m)$ define from Theorem 3

$$
F(n, 0)=F(0, m)=0 \quad \text { and } \quad U(n-1, m-1)=3^{n-1} 2^{m} \sum_{l=0}^{m} \sum_{k=0}^{n} \frac{1}{2^{l} l ! k !}(n-k)(m-l) F(n-k, m-l) .
$$

Using equation (3), we get

$$
u(x, t)=1+x+t+x t+\frac{1}{2 !} t^{2}+\frac{1}{2 !} x^{2}+\frac{1}{1 ! 2 !} x t^{2}+\frac{1}{2 ! 1 !} x^{2} t+\frac{1}{2 ! 2 !} x^{2} t^{2}+\ldots
$$

which is the Taylor series of function $u(x, t)=e^{x+t}$ and exact solution of Example 2.

Example 3. Consider the following two-dimensional integral equation with proportional delays

$$
\left.u(x, t)=\cos (x+t)-8 \sin \frac{x}{12} \sin \frac{t}{4}\right)+\frac{1}{\cos \left(\frac{x}{12}+\frac{t}{4}\right)} \int_{0}^{t / 2 x / 3} \int_{0}^{x} u\left(\frac{y}{2}, z\right) d z d y .
$$

The exact solution of the problem is $u(x, t)=\cos (x+t)$.

Now, using differential transform of last equation, we have

$$
U(n, m)=F(n, m)+\frac{1}{n ! m !} \cos \frac{\pi}{2}(n+m)-\frac{8}{n ! m ! 12^{n} 4^{m}} \sin \frac{n \pi}{2} \sin \frac{m \pi}{2}
$$

where $F(n, m)$ define from following relations:

$$
\begin{aligned}
F(n, 0) & =F(0, m)=0 \\
\sum_{l=0}^{m} \sum_{k=0}^{n} \frac{\cos \frac{\pi}{2}(n+m)}{k ! l ! 12^{k} 4^{l}} F(n-k, m-l) & =\frac{1}{n m 3^{n} 2^{n+m-1}} U(n-1, m-1) .
\end{aligned}
$$

Using (5) we have the following relations:

$$
\begin{array}{rl}
U(0,0)=F(0,0)+1-0 & U(2,0)=F(2,0)-\frac{1}{2}-0 \\
U(0,1)=F(0,1)+0-0 & U(1,1)=F(1,2)+0-0 \\
U(1,0)=F(1,0)+0-0 & U(2,1)=F(2,1)+0-0 \\
U(1,1)=F(1,1)-1-\frac{8}{12 * 4} & U(2,2)=F(2,2)+\frac{1}{4}-0 \\
U(0,2)=F(0,2)-\frac{1}{2}-0 & \ldots
\end{array}
$$

Using relations (6-7) from Theorem 3 and equation (3), we gain

$$
u(x, t)=1-x t-\frac{1}{2} t^{2}-\frac{1}{2} x^{2}+\frac{1}{4} x^{2} t^{2}+\ldots
$$

which is the Taylor series of exact solution of Example 3. 


\section{Conclusions}

In this study, the differential transform method has been presented for solving two-dimensional Volterra integral equations. A new theorem is introduced with its proof, and as application some examples are carried out. If solution of equation is polinomial function, then method gives the exact solution, in other cases, the rapidly converging series solution.

\section{Competing interests}

The authors declare that they have no competing interests.

\section{Authors' contributions}

All authors have contributed to all parts of the article. All authors read and approved the final manuscript.

\section{References}

[1] V. Volterra, Sopra alcune questioni di inversione di integrali definite, Ann.Mat. Pura Appl., (2) 25, 139-178, 1897.

[2] K.L. Cooke, J.A. Yorke, Some equations modelling growth processes and epidemics, Math. Biosci., 16, 75-101, 1973.

[3] P. Waltham, Deterministic Threshold models in the Theory of Epidemics, Lecture Notes in Biomath., Vol. 1, Springer-Verlag (Berlin-Heidelberg), 1974.

[4] H.L. Smith, On periodic solutions of a delay integral equation modelling epidemics, J. Math. Biol., 4, 69-80, 1977.

[5] S. Busenberg, K.L. Cooke, The effect of integral conditions in certain equations modelling epidemics and population growth, J. Math. Biol., 10, 13-32, 1980.

[6] J.A.J. Metz, O. Diekmann, The Dynamics of Physiologically Structured Populations, Lecture Notes in Biomath., Vol. 68, SpringerVerlag (Berlin- Heidelberg), 1986.

[7] H.W. Hethcote, P. van den Driessche, Two SIS epidemiologic models with delays, J. Math. Biol., 40, 3-26, 2000.

[8] F. Brauer, P. van den Driessche, Some directions for mathematical epidemiology, in Dynamical Systems and Their Applications in Biology, Fields Institute Communications, Vol. 36, American Mathematical Society (Providence), 95-112, 2003.

[9] H. Brunner, On the discretization of differential and Volterra integral equations with variable delay, BIT 37, 1-12, 1997.

[10] N.Takama,Y.Muroyaand, E.Ishiwata, On the discretization of differential and Volterra integral equations with variable delay, BIT37,1-12, 2000.

[11] C.J.Zhangand, S.Vandewalle, On the attainable order of collocation methods for the delay differential equations with proportional delay, BIT40, 374-394, 2008.

[12] A.Bellen, Stability criteria for exatand discrete solutions of neutral multidelay-integro-differential equations, Adv.Comput.Math., 28, 383-399, 2002.

[13] Ş.Yüzbaş1, Laguerre approach for solving pantograph-type Volterra integro-differential equations, Applied Mathematics and Computation, 232, 1183-1199, 2014.

[14] N. Şahin, Ş. Yüzbaşı,M. Gülsu, A collocation approach for solving systems of linear Volterra integral equations with variable coefficients, Computers and Mathematics with Applications, 62, 755-769, 2011.

[15] E.Yusufoğlu, A homotopy perturbation algorithm to solve a system of Fredholm-Volterra type integral equations, Mathematical and Computer Modelling 47 (2008) 1099-1107.

[16] J.Saberi-Nadjafi, M.Tamamgar, The variational iteration method: A highly promising method for solving the system of integrodifferential equations, Computers and Mathematics with Applications 56 (2008) 346-351.

[17] K. Maleknejad, M.Tavassoli Kajani, Solving linear integro-differential equation system by Galerkin methods with hybrid functions, Applied Mathematics and Computation 159 (2004) 603-612. 
[18] J. Biazar, E. Babolian, R. Islam, Solution of a system of Volterra integral equations of the first kind by Adomian method, Applied Mathematics and Computation 139, 249-258 (2003).

[19] G. E. Pukhov, Differential transforms and circuit theory, Int. J. Circ. Theor. App. 10, 265, 1982.

[20] J. K. Zhou, Differential transformation and its applications for electrical circuits, in Chinese, Huarjung University Press, Wuuhahn, China, 1986.

[21] A. Tari, M.Y. Rahimi, S. Shahmorad, F. Talati, Solving a class of two-dimensional linear and nonlinear Volterra integral equations by the differential transform method, Journal of Computational and Applied Mathematics, 228, 70-76, 2009.

[22] A. Tari, S. Shahmorad, Differential transform method for the system of two-dimensional nonlinear Volterra integro-differential equations, Computers and Mathematics with Applications, 61, 2621-2629, 2011.

[23] Bongsoo Jang, Comments on "Solving a class of two-dimensional linear and nonlinear Volterra integral equations by the differential transform method", Journal of Computational and Applied Mathematics, 233, 224-230, 2009.

[24] R.Abazari, M.Ganji, Extended two-dimensional DTM and its application on nonlinear PDEs with proportional delay, International Journal of Computer Mathematics, Vol. 88, No. 8, 1749-1762, 2011. 